\title{
ChemComm
}

Can aromaticity be a kinetic trap? Example of mechanically interlocked aromatic [2-5]catenanes built of cyclo[18]carbon

\begin{tabular}{|r|l|}
\hline Journal: & ChemComm \\
\hline Manuscript ID & CC-COM-12-2019-009483.R1 \\
\hline Article Type: & Communication \\
\hline & \\
\end{tabular}

SCHOLARONE ${ }^{\text {M }}$

Manuscripts 


\section{Can aromaticity be a kinetic trap? Example of mechanically interlocked aromatic [2-5]catenanes built of cyclo[18]carbon}

Received 00th January 20xx,

Nikita Fedik ${ }^{a}$, Maksim Kulichenko ${ }^{a}$, Dmitriy Steglenko ${ }^{b}$, Alexander I. Boldyrev *a,b

Accepted 00th January 20xx

DOI: $10.1039 / \times 0 \times x 00000 x$

Unusual stability of cyclo[18]carbon arising from its aromaticity might be used to provide kinetic trapping needed in design of interlocked systems. Kinetic barrier separating interlocked rings and chemically bonded complex is about $30 \mathrm{kcal} / \mathrm{mol}$. In addition, rings can slide freely which is promising property towards design of molecular gears and motors.

The immanent propensity of human nature to seek for aesthetically pleasant, symmetric, unusual patterns and topologies led to many serendipities, especially, in the field of organic chemistry. After centennial legacy of perfectly hexagonal benzene, $20^{\text {th }}$ century was enlightened by game changing discoveries of new carbon allotropes including fullerene ${ }^{1-3}$ having perfect soccer ball $l_{h}$ symmetry and honeycomb graphene ${ }^{4-6}$. One of the most prominent recent discoveries is the synthesis of cyclo[18]carbon ${ }^{7}$ (furtherly referred simply as $\mathrm{C}_{18}$ ) - a new carbon allotrope, which takes honourable place among 2019 year molecules, according to C\&EN ranking ${ }^{8}$. Ground state structure of $\mathrm{C}_{18}$ has been a topic of many theoretical debates throughout decades ${ }^{9-15}$. Experimental characterization of structure confirmed its unusual polyynic structure belonging to $C_{9 h}$ point group and having alternating single and triple bonds. For a member of cyclo[ $4 n+2]$ carbone family satisfying Hückel's rule $4 n+2$, pronounced bond alternation looks very surprising. Moreover, it is considered to be doubly aromatic having 2 mutually orthogonal sets of $9 \pi$-orbitals ${ }^{14,16}$. Intuitively, aromaticity is inherently associated with the equalization of bonds due to formation of conjugated $\pi$-system, however, cumulenic structure was shown to be a transition state (TS) between true alternating ground states $^{14}$ which structure was finally elucidated experimentally ${ }^{7}$. Such alternation was rationalized in terms of second order Jahn-Teller's effect ${ }^{14,16}$. Interestingly, it

\footnotetext{
a. Department of Chemistry and Biochemistry, Utah State University, 0300 Old Main Hill, Logan, UT, 84322-0300, USA.

b. Institute of Physical and Organic Chemistry, Southern Federal University, Rostovon-Don 344090, Russia

*a.i.boldyrev@usu.edu

Electronic Supplementary Information (ESI) available: [discussion about theoretical methods and Cartesian coordinates of all structures]. See DOI: 10.1039/x0xx00000x
}

was predicted about two decades ago by Saito and co-workers prior to experimental confirmation ${ }^{16}$.

Non-trivial ring-shaped structure of new allotrope alongside with its unprecedent stability arising from double aromaticity ${ }^{14}$ makes $\mathrm{C}_{18}$ one of the most promising candidates for the mechanically interlocked systems ${ }^{17-21}$. Such molecular ensembles are also referred as kinetically trapped indicating that such stoichiometry might not be a global minimum on PES, however, the interlocked state is preserved due to high activation energy required to break the cyclic structures or to bring monomers together to form true chemical bonds. For many years mechanically interlocked systems and, especially, catenanes have been attracting close attention from scientific community. Low-rotational barriers in some catenanes make them prospective building blocks for molecular electronics ${ }^{17,22}$ while their unusual shape might be used to host bioactive molecules and design of drug-delivery vectors ${ }^{23}$. Moreover, their potential might be realized in molecular sensors and catalysis ${ }^{24}$ owing to their capability to host and efficiently orientate transition metals ${ }^{25}$.

For many years catenanes and other topologies were elusive in real synthesis because of the low probability of formation upon statistical approach. However, recent advances in polycatenanes synthesis, especially template-directed approach, allowing preorientation of fragments opened the gate to obtain experimentally wide range of locked systems. This strategy was successfully expanded to poly[n]catenanes and so far the cutting edge example is the targeted synthesis of poly[130]catenane ${ }^{18}$. Another impressive example of allbenzene catenane suggests new synthetic strategy for catenane without heteroatoms which was impossible for a long time ${ }^{26}$. Noticeably, this interlocked system is also listed in a prestigious ranking of molecules of 2019 year $^{8}$.

Following recent success both in $\mathrm{C}_{18}$ characterization and design of [n]catenanes, in this study we would like to address the possibility of existence of $\mathrm{C}_{18}$-based [n]catenanes and their kinetic stability. For the successful design - either experimental 

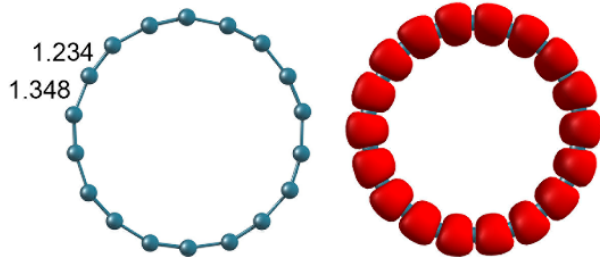

C9n

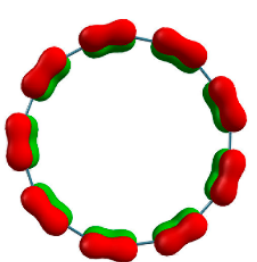

$18 \times 2 \mathrm{c}-2 \mathrm{e} \mathrm{C}-\mathrm{C} \sigma$-bonds $\mathrm{ON}=1.99|\mathrm{e}|$

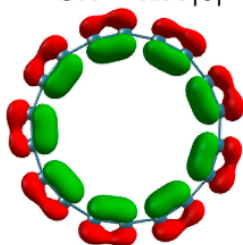

2 orthogonal sets of $9 \times 2 \mathrm{c}-2 \mathrm{e} \mathrm{C}-\mathrm{C} \pi$-bonds $\mathrm{ON}=1.82 \mid \mathrm{e}$

Figure 1. Bonding pattern of sole $C_{18}$ ring. Hereinafter bond lengths are in $\AA$. ON stands for occupation number.

or theoretical - two main criteria should be satisfied. First, to form a true catenane structures should interact only "mechanically", so by that we mean that no new chemical ('electronic') bonds are formed upon rings closing, thus structural features of monomers are mostly preserved. Second, the kinetic barrier, separating mechanically interacting complex and chemically bonded one, should be significant to provide the state known as kinetic trap. The latter condition is necessary for the system existence because, obviously, catenanes most likely are not global minima on PES, thus, only high activation barrier can preserve their state. While aromaticity is inherently associated with unusual thermodynamic stability of molecules $^{27,28}$ and clusters ${ }^{29-35}$, we aim to show that it could also be used for kinetic trapping and, hence, targeted design of mechanically interlocked systems.

Although computational study of $\mathrm{C}_{18}$ and its derivatives looks straight forward, it is in fact full of pitfalls. For the selection of the level of theory we would like to refer to the recent study of Sundholm and co-authors in which this topic is discussed exhaustively ${ }^{14}$. It is remarkable that many standard time-tested functionals (such as B3LYP or PBEO) fail to reproduce experimentally observed polyynic geometry of $C_{18}$ which is explained by insufficient treatment of Hartree-Fock exchange ${ }^{14}$. All systems considered in this work were studied at the recommended M06-2X/6-31+G*36,37 level of theory which correctly predicts the polyynic system to be energy minimum. In addition, to treat interatomic interactions crucial for mechanical bonds Grimme's dispersion correction (GD3) was employed in all calculations ${ }^{38}$.

Let us start with the bonding of $\mathrm{C}_{18}$ ring which is a monomer of the suggested [n]catenanes. Bonding picture was deciphered using AdNDP method ${ }^{39-45}$, flexible tool based on NBO analysis ${ }^{46,47}$. As expected, frame of $18 \sigma$-bonds was found which is accomplished by two mutually orthogonal sets of $9 \pi$-bonds (Fig 1). This picture is in full agreement with the experimental observation and CCSD calculations ${ }^{10,14,16}$.

Simplest precursor of poly[n]catenanes is [2]catetane constructed of two $\mathrm{C}_{18}$ rings. Such interesting molecular

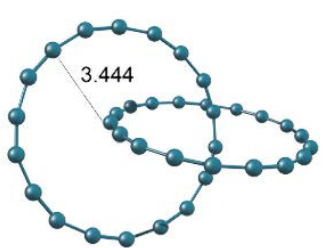

$C_{1}$

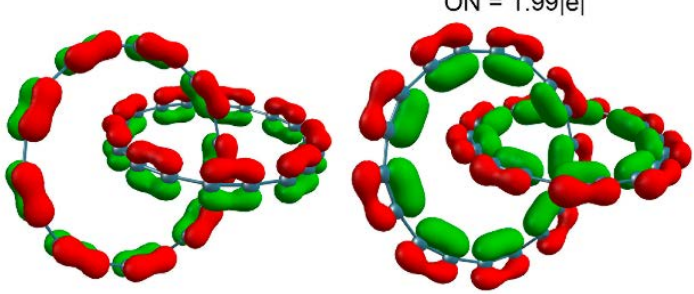

$2 \times 2$ orthogonal sets of $9 \times 2 \mathrm{c}-2 \mathrm{e} \mathrm{C}-\mathrm{C} \pi$-bonds $\mathrm{ON}=1.82|\mathrm{e}|$

Figure 2. Bonding pattern of [2]catenane formed by two $C_{18}$ rings. Closest distance between two atoms from different rings is shown in $\AA$.

ensembles are also known as a Hopf link ${ }^{19}$. Recovered bonding pattern of [2]catetane perfectly resembles the one obtained for the monomer, therefore, the first criterion of mechanical bond is fully satisfied (Fig 2). Moreover, ONs remain the same indicating complete absence of electron transfer. In addition, the distances between two $\mathrm{C}$ atoms belonging to different rings are around $3.5 \AA$ which even intuitively means absence of chemical bond.

Some of previously reported Hopf links can easily slide along each other ${ }^{48}$. This is true in case of considered [2]catenane. Calculated barrier of rotation is less than $0.01 \mathrm{kcal} / \mathrm{mol}$ meaning free rotation of rings along each other whereas energy difference between rotamers is within $0.2 \mathrm{kcal} / \mathrm{mol}$. Obviously, such rotation is perpetually induced by thermal motion at room temperature, which is a valuable property for molecular gears and motors ${ }^{49-52}$.

While mechanical character of inter-ring bond in [2]catenane is confirmed, it is necessary to investigate whether such structure is kinetically trapped or not. To enlighten that issue we studied kinetic stability as a reaction pathway between a [2]catenane and chemically bonded complex (Fig 3). Based on the energetic profile of the reaction we can clearly see that suggested arrangement is, in fact, kinetically trapped because the kinetic barrier separating mechanically interacting rings and complex is $31.1 \mathrm{kcal} / \mathrm{mol}$. Moreover, Pretzel-like adduct is less stable than Hopf link by $21.1 \mathrm{kcal} / \mathrm{mol}$ since initial aromaticity is distorted. Both TS and adduct exhibit predictable RHF > UHF wave function instability owing to closed-shell singlet character. Therefore, reaction pathway was studied using brokensymmetry (BS) UDFT-approach which was shown previously to be accurate for large organic molecules ${ }^{53-55}$. Furtherly, biradical open-shell singlet character of TS adduct was confirmed by $\operatorname{CASSCF}(8,8)$ method. However, one should keep in mind that the barrier value provided is a BS-UDFT estimation and more accurate results might be obtained only by demanding multireference methods dressed by dynamic correlation. Moreover, in the absence of any experimental data it is impossible to say how close DFT results are to the true energetics but, considering 


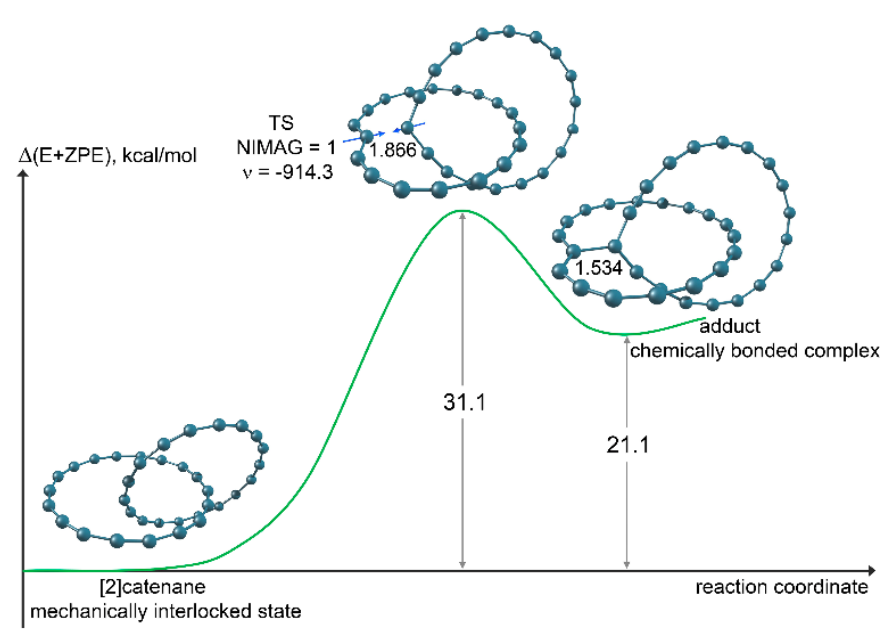

Figure 3. Energy profile of the formation of chemical complex from [2]catenane, BSUM06-2X/6-31+G*.

previous successful applications ${ }^{53-55}$ of BS-UDFT approach, we believe that the studied pathway should be correct. All relevant computational details are placed in ESI. Consideration of further transformation of bonded cycle to possibly more stable structures are beyond the scope of our study because it does not provide any additional insight on kinetic stability which might be assessed based on first (presumably rate-limited) barrier. From the barrier obtained it is already obvious that the system under consideration is kinetically trapped as any catenane should be. To leave the kinetic trap, significant amount of energy (no less than $\sim 30 \mathrm{kcal} / \mathrm{mol}$ ) has to be provided which might be explained by the aromatic character of individual rings. Low rotational barrier implies that full PES of such [2]catenane is extremely complex and accounts for large number of fully or nearly energetically degenerate rotamers. Therefore, we believe that in addition to reaction pathway found, many other could coexist and we expect them to be around the same unfavourable energetics.

As for the poly[n]catenanes, we studied systems up to 5 rings and all of them are revealed to be local minima (Fig 4A-C). If the latest arrangement is synthesized, it might earn an honourable place among other molecular Olympic rings such as olympicene $\mathrm{e}^{56}$ and olympiadane ${ }^{57}$.

Stability trend clearly shows that we should expect longer chains of poly[n]catenanes to be stable as well. It might be interesting to investigate such chains using periodic boundary conditions (PBC) implemented in solid-state codes. However, such approach faces many challenges. As we already discussed, chains are very flexible, therefore, plenty of rotamers coexists on the PES. Second, the large unit cell should be considered to employ PBC using exact exchange treatment ${ }^{14}$ (by pure HF or any hybrid functional). Such approach demands enormous computational resources without any guarantee that even good guess would converge to the energy-minimum rotamer.

Noticeably, that so far $\mathrm{C}_{18}$ was obtained at $5 \mathrm{~K}$, and its stability at higher temperature remains unclear. Previously, it was shown that carbon rings can coalesce forming fullerenes or other derivatives 7,58 at high temperatures, and that might be true for [n]catenanes as well. Hence, we expect that suggested

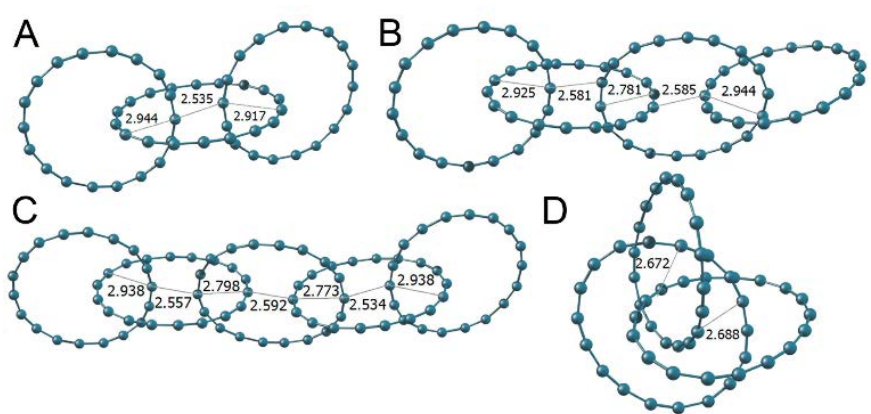

Figure 4. A-C - [3-5]catenanes, respectively. D - Borromean rings built of three $C_{18}$ monomers. Nearest distances between different rings are shown.

kinetic trap works under low temperatures. Calculated pathway was discussed in terms of E+ZPE, thus, in temperatureindependent fashion. To estimate the temperature range in which kinetic trap works, one need to perform molecular dynamics simulations which are very challenging by the reasons described above.

Lastly, we would like to consider another peculiar topology known as Borromean rings $^{19}$ consisting of three mutually interlocked rings (Fig 4D). As it turned out, this system is also an energy minimum in which there are no chemical bonds between monomers (Fig S1). Remarkably, $\mathrm{C}_{18}$ rings can adjust their shapes to host other monomers. For example, individual $\mathrm{C}_{18}$-monomers of Borromean rings as well as in intermediate ring in poly[3-5]catenanes take oval-like shape to reduce stereochemical "pressure". Such noticeable adaptability has a great potential toward the design of host-guest systems.

To briefly recap, we showed that [2-5]catenanes and Borromean rings built of $\mathrm{C}_{18}$ rings exhibit essential stability when mechanically interlocked. Our conclusions are based on the combined study of chemical bonding and the kinetics of chemical complex formation from mechanically interacting rings. Formation of interlocked systems does not disturb initial aromaticity of individual rings, therefore, serving as a kinetic trap which estimated barrier is about $30 \mathrm{kcal} / \mathrm{mol}$.

We limited our consideration up to [5]catenane but, based on the obtained results, we expect poly[n]catenanes to be stable as well because they are constructed by the same principle. Taking into account brilliant successes of synthetic approaches to poly[n]catenanes ${ }^{18}$, new report on all-benzene catenane ${ }^{26}$, and range of prospective applications we believe that systems under consideration are not elusive prognostications but promising candidates for the supramolecular synthesis.

\section{Conflicts of interest}

There are no conflicts to declare.

\section{Acknowledgments}

The work was supported by the Russian Government grant by decree N 220 (agreement № 14.Y26.31.0016) and by the USA National Science Foundation (grant CHEM-1664379). The 
support and resources from the Center for High Performance Computing at the University of Utah are gratefully acknowledged.

\section{Notes and references}

1 M. Prato, J. Mater. Chem., 1997, 7, 1097-1109.

2 V. Georgakilas, J. A. Perman, J. Tucek and R. Zboril, Chem. Rev., 2015, 115, 4744-4822.

3 A. Zieleniewska, F. Lodermeyer, A. Roth and D. M. Guldi, Chem. Soc. Rev., 2018, 47, 702-714.

4 M. Coroş, F. Pogăcean, L. Măgeruşan, C. Socaci and S. Pruneanu, Front. Mater. Sci., 2019, 13, 23-32.

5 K. S. Novoselov, A. K. Geim, S. V Morozov, D. Jiang, Y. Zhang, S. V. Dubonos, I. V Grigorieva and A. A. Firsov, Science, 2004, 5, 1-12.

6 A. Hirsch, Nat. Mater., 2010, 9, 868-871.

7 K. Kaiser, L. M. Scriven, F. Schulz, P. Gawel, L. Gross and H. L. Anderson, Science, 2019, 365, 1299-1301.

8 https://cen.acs.org/synthesis/CENs-molecules-year2019/97/i48 (accesed on 12.5.18).

9 K. Remya and C. H. Suresh, RSC Adv., 2016, 6, 44261-44271.

10 S. Arulmozhiraja and T. Ohno, J. Chem. Phys., 2008, 128 114301.

11 R. S. Zhang and J. W. Jiang, Front. Phys., 2019, 14, 13401.

12 M. Feyereisen, M. Gutowski, J. Simons and J. Almlöf, J. Chem. Phys., 1992, 96, 2926-2932.

13 V. Parasuk, J. Almlöf and M. W. Feyereisen, J. Am. Chem. Soc., 1991, 113, 1049-1050.

14 G. V. Baryshnikov, R. R. Valiev, A. V. Kuklin, D. Sundholm and H. Ågren, J. Phys. Chem. Lett., 2019, 6701-6705.

15 J. M. L. Martin, J. El-Yazal, J.-P. Franqois, Chem. Phys. Lett., 1995, 242, 570-579.

16 M. Saito and Y. Okamoto, Phys. Rev. B - Condens. Matter Mater. Phys., 1999, 60, 8939-8942.

17 D. Sluysmans and J. F. Stoddart, Trends Chem., 2019, 1, 185197.

18 Q. Wu, P. M. Rauscher, X. Lang, R. J. Wojtecki, J. J. De Pablo, M. J. A. Hore and S. J. Rowan, Science, 2017, 358, 14341439.

19 H. Y. Au-Yeung, C. C. Yee, A. W. Hung Ng and K. Hu, Inorg Chem., 2018, 57, 3475-3485.

20 S. Mena-Hernando and E. M. Pérez, Chem. Soc. Rev., 2019, 48, 5016-5032.

21 N. H. Evans and P. D. Beer, Chem. Soc. Rev., 2014, 43, 46584683.

22 A. Coskun, J. M. Spruell, G. Barin, W. R. Dichtel, A. H. Flood Y. Y. Botros and J. F. Stoddart, Chem. Soc. Rev., 2012, 41, 4827-4859.

23 N. Pairault, R. Barat, I. Tranoy-Opalinski, B. Renoux, M. Thomas and S. Papot, Comptes Rendus Chim., 2016, 19, 103112.

24 L. Hu, C. H. Lu and I. Willner, Nano Lett., 2015, 15, 20992103.

25 B. Korybut-Daszkiewicz, A. Więckowska, R. Bilewicz, S. Domagala and K. Woźniak, J. Am. Chem. Soc., 2001, 123 9356-9366.

26 Y. Segawa, M. Kuwayama, Y. Hijikata, M. Fushimi, N. Kubota and K. Itami, Science, 2019, 276, 272-276.

27 N. Fedik and A. I. Boldyrev, J. Phys. Chem. A, 2018, 122, $8585-8590$

28 S. Furukawa, M. Fujita, Y. Kanatomi, M. Minoura, M. Hatanaka, K. Morokuma, K. Ishimura and M. Saito, Commun. Chem., 2018, 1, 60.

29 N. Fedik, A.I. Boldyrev, A. Mũnoz-Castro, Phys. Chem. Chem Phys., 2019, 21, 25215-25219.
30 N. Fedik, M. Kulichenko and A. I. Boldyrev, Chem. Phys., 2019, 522, 134-137.

31 Z. Chen and R. B. King, Chem. Rev., 2005, 105, 3613-3642.

32 R. B. King, Chem. Rev., 2001, 101, 1119-1152.

33 O. A. Gapurenko, R. M. Minyaev, N. S. Fedik, V. V. Koval, A. I. Boldyrev and V. I. Minkin, Struct. Chem., 2019, 30, 805-814.

34 M. Kulichenko, N. Fedik, A. Mũnoz-Castro and A. I. Boldyrev, Chem. - A Eur. J., doi.org/10.1002/chem.201905088

35 C. Liu, N. V. Tkachenko, I. A. Popov, N. Fedik, X. Min, C-Q. Xu, J. Li, J. E. McGrady, A. I. Boldyrev and Z.-M. Sun, Angew. Chemie - Int. Ed., 2019, 58, 8367-8371.

36 Y. Zhao and D. G. Truhlar, Theor. Chem. Acc., 2008, 120, 215241.

37 R. Ditchfield, W. J. Hehre and J. A. Pople, J. Chem. Phys., 1971, 54, 724-728.

38 S. Grimme, J. Antony, S. Ehrlich and H. Krieg, J. Chem. Phys., 2010, 132, 154104

39 D. Y. Zubarev and A. I. Boldyrev, Phys. Chem. Chem. Phys., 2008, 10, 5207-5217.

40 D. Y. Zubarev and A. I. Boldyrev, J. Org. Chem., 2008, 73, 9251-9258.

41 M. Kulichenko, N. Fedik, K. V. Bozhenko and A. I. Boldyrev, J. Phys. Chem. B, 2019, 123, 4065-4069.

42 M. Kulichenko, N. Fedik, K. V. Bozhenko and A. I. Boldyrev, Chem. - A Eur. J., 2019, 25, 5311-5315.

43 G. Liu, N. Fedik, C. Martinez-Martinez, S. M. Ciborowski, X. Zhang, A.I. Boldyrev and K. H. Bowen, Angew. Chemie - Int Ed., 2019, 58, 13789-13793.

44 N. V. Tkachenko, D. Steglenko, N. Fedik, N. M. Boldyreva, R. M. Minyaev, V. I. Minkin, A. I. Boldyrev, Phys. Chem. Chem. Phys., 2019, 21, 19764-19771.

45 J. Czekner, L. F. Cheung, G. S. Kocheril, M. Kulichenko, A. I. Boldyrev, L.-S. Wang, Angew. Chemie - Int. Ed, 2019, 58, 8877-8881.

46 E. D. Glendening, J. K. Badenhoop, A. E. Reed, J. E. Carpenter J. A. Bohmann, C. M. Morales, C. R. Landis and F. Weinhold, NBO 6.0, Theoretical Chemistry Institute, University of Wisconsin, Madison, 2013.

47 J. P. Foster and F. Weinhold, J. Am. Chem. Soc., 1980, 102, 7211-7218.

48 I. R. Fernando, M. Frasconi, Y. Wu, W. G. Liu, M. R. Wasielewski, W. A. Goddard and J. F. Stoddart, J. Am. Chem Soc., 2016, 138, 10214-10225.

49 E. E. Burnell, C. A. De Lange and W. L. Meerts, J. Chem. Phys., 2016, 145, 091101

50 M. Nakamura, K. Kishimoto, Y. Kobori, T. Abe, K. Yoza and K. Kobayashi, J. Am. Chem. Soc., 2016, 138, 12564-12577.

51 R. Zhao, Y. L. Zhao, F. Qi, K. E. Hermann, R. Q. Zhang and M. A. Van Hove, ACS Nano, 2018, 12, 3020-3029.

52 J. F. Stoddart, Angew. Chemie - Int. Ed., 2017, 56, 1109411125.

53 J. Gräfenstein, E. Kraka, M. Filatov and D. Cremer, Int. J. Mol. Sci., 2002, 3, 360-394.

54 M. Bendikov, H. M. Duong, K. Starkey, K. N. Houk, E. A. Carter and F. Wudl, J. Am. Chem. Soc., 2004, 126, 74167417.

55 A.I. Krylov, The Quantum Chemistry of Open-Shell Species. In Reviews in Computational Chemistry (eds A.L. Parrill and K.B. Lipkowitz), 2017.

56 A. Mistry, B. Moreton, B. Schuler, F. Mohn, G. Meyer, L. Gross, A. Williams, P. Scott, G. Costantini and D. J. Fox, Chem - A Eur. J., 2015, 21, 2011-2018.

57 D. B. Amabilino, P. R. Ashton, A. S. Reder, N. Spencer and J. F. Stoddart, Angew. Chemie Int. Ed. English, 1994, 33, 12861290.

58 G. von Helden, N. G. Gotts, M. T. Bowers, Nature, 1993, 363 60-63. 